\title{
Left atrial stiffness: a novel marker of hypertension-mediated organ damage on the horizon?
}

\author{
Cesare Cuspidi ${ }^{1,2} \cdot$ Roberto Giovannini $^{1} \cdot$ Marijana Tadic $^{3}$
}

Received: 12 October 2020 / Revised: 20 October 2020 / Accepted: 22 October 2020 / Published online: 1 December 2020

(c) The Japanese Society of Hypertension 2020

Hypertension-mediated subclinical organ damage is currently regarded as an intermediate step in the continuum of cardiovascular disease and a strong, independent predictor of cardiovascular events and all-cause mortality. Hypertension adversely affects cardiac structure and function by inducing a variety of alterations, including myocyte hypertrophy, fibrosis, impaired left ventricular (LV) contractility and relaxation, left atrium enlargement (LAE), and aortic dilatation. Although LVH is recognized as the cardinal biomarker of target cardiac damage, the clinical and prognostic significance of LA has progressively gained attention over the past few decades. However, research on the role of the LA in hypertensive heart disease has long been conditioned by several difficulties in evaluating the structural and functional characteristics of this chamber.

Over 50 years ago, a pioneering study by Tarazi et al., conducted on 76 hypertensive patients and 76 normal subjects matched for age and sex, showed for the first time that LA abnormality is a common electrocardiographic finding in hypertension not necessarily associated with LV hypertrophy (LVH) or heart failure [1]. LA abnormality, as assessed by four different criteria, was present in 59\% of hypertensive patients and in only $5 \%$ of apparently healthy controls. The authors suggested that these LA alterations in the hypertensive setting could indicate active atrial participation in ventricular function or reflect diminished ventricular compliance even in the absence of electrocardiographic signs of LVH. Approximately 12 years later, a further step in the study of LA in hypertensive heart disease was taken with the affirmation of echocardiography as a reference imaging technique.

Cesare Cuspidi

cesare.cuspidi@unimib.it

1 Department of Medicine and Surgery, University of MilanoBicocca, Milano, Italy

2 Istituto Auxologico Italiano, Milano, Italy

3 Department of Cardiology, University Hospital "Dr. Dragisa Misovic-Dedinje", Belgrade, Serbia
A seminal study by Miller et al. including a total of 26 patients (10 controls and 14 hypertensive patients) in sinus rhythm, with normal coronary arteriography; normal LV volumes, function, and LV mass; absence of valvular disease; electrocardiographic signs of LA abnormality and LVH documented that LAE, as assessed by echocardiography, is actually an early sign of hypertensive heart disease [2]. In fact, the mean LA diameter was $3.46 \mathrm{~cm}$ in normal individuals versus $4.04 \mathrm{~cm}$ in hypertensive patients $(p<0.01)$, and the difference persisted even after indexing by body surface area ( 2.18 versus $1.88 \mathrm{~cm} / \mathrm{m}^{2}, p<0.05$ ).

Currently, a large body of evidence supports the view that in hypertensive patients, LAE, phenotyped by measuring the diameter or more precisely, the volume with echocardiography, is a reliable marker of chronically elevated LV filling pressure and diastolic dysfunction. The increase in LA size is considered a compensatory mechanism aimed at counterbalancing the impairment of LV compliance and progressive deterioration of $\mathrm{LV}$ diastolic dysfunction in the hypertrophied ventricle. However, it must be recognized, in line with the results of early studies, that LAE may develop before hypertensive LVH is manifested.

Among 1785 individuals representative of the Italian general population for sex and age (25-74 years) participating in the Pressioni Monitorate e Loro Associazioni study, an isolated LAE was by no means rare; however, because it affected approximately a quarter $(24.7 \%)$ of the individuals with any cardiac structure alteration, it amounted to $4.7 \%$ of the sample [3].

Several cross-sectional and longitudinal studies have reported that in the hypertensive setting, the likelihood of having LAE is strongly influenced by additional risk factors, such as old age, diabetes, low HDL cholesterol, obesity, sleep apnea syndrome, and LVH. For instance, the Losartan Intervention For Endpoint reduction in hypertension study has demonstrated that a larger LA diameter is independently related to a variety of unfavorable covariates, including higher systolic blood pressure, body mass index, LV mass, age, atrial fibrillation, and mitral regurgitation [4]. 
The prevalence of LAE in hypertension has been the topic of numerous individual studies and their metaanalysis. In a study by our group including 15 studies totaling 10,141 untreated and treated hypertensive patients, the prevalence of LAE in the pooled population was $32 \%$, without differences between men and women [5]. Of note, data provided by ten studies ( $n=9354$ patients) showed that LVH was significantly more frequent in patients with LAE $(68 \%)$ than in their counterparts without it $(41 \%)$.

Regarding the prognostic significance of LAE, longitudinal studies have consistently demonstrated that this echocardiographic phenotype is an independent predictor of stroke, atrial fibrillation, myocardial infarction, heart failure, cardiovascular death, and all-cause mortality in hypertensive cohorts, general population samples, elderly subjects, and patients with chronic kidney disease. This has been shown to occur when the enlargement is detected either via LA diameter (an easily measurable with satisfactory reproducibility) or via a more comprehensive assessment based on the LA volume. Unfortunately, compared to the large amount of clinical and prognostic data accumulated on atrial size, the analysis of LA function and inherent clinical implications evolved significantly more slowly due to its complexity (the need for a set of parameters) and the lack of adequate noninvasive diagnostic techniques. The LA serves as (i) a conduit for the transfer of blood from pulmonary veins into the LV; (ii) a reservoir for blood storage during LV contraction and isovolumetric relaxation; and (iii) a contractile chamber to complete LV filling during end-diastole [6].

In the last decade, the introduction of strain imaging in echocardiography significantly improved knowledge about cardiac mechanics and provided better and more detailed insight into LA mechanics in several clinical settings, including systemic hypertension [7]. This is because strain evaluation provides even more information about each phase of cardiac diastole and therefore LA functions.

In the research area aimed at identifying new markers of subclinical cardiac organ damage in uncomplicated hypertensive patients via updated echocardiographic tools, the study by Zhao et al., published in this issue of the Journal, adds a new piece of information [8]. Starting from the hypothesis that abnormalities in LA mechanics assessed by strain and strain rate imaging using two-dimensional speckle-tracking echocardiography may precede the onset of conventional and prognostically validated markers of cardiac organ damage such as LAE and LVH, the authors investigated LA function in 328 individuals categorized into four groups: (I) healthy normotensive subjects ( $n=42)$, (II) hypertensive patients without LAE and LVH $(n=79)$, (III) hypertensive patients with normal LV mass and LAE $(n=$ 92), and (IV) hypertensive patients with LVH $(n=115)$.

All patients were admitted to a cardiology department and underwent investigations to exclude secondary hypertension, coronary heart disease, cardiomyopathy, and significant valve disease. Although many of them were treated for hypertension (mainly with calcium channel blockers, less frequently with angiotensin-converting enzyme inhibitors or angiotensin II antagonists and only rarely with diuretics), they had quite high clinical blood pressure values (i.e., on average $\sim 160-\mathrm{mmHg}$ systolic blood pressure and $>90-\mathrm{mmHg}$ diastolic blood pressure). The diagnostic criteria used for the phenotyping of LAE and LVH were $28 \mathrm{ml} / \mathrm{m}^{2}$ for both sexes and $95 \mathrm{~g} / \mathrm{m}^{2}$ in women and $115 \mathrm{~g} / \mathrm{m}^{2}$ in men, respectively. In the entire population, the prevalence of LAE and LVH was 32 and $49 \%$, respectively. The following LA mechanics parameters were taken into account: reservoir strain and strain rate, conduit strain and strain rate, and LA booster strain and strain rate. Of note, in addition to these established indexes, the myocardial mechanics LA stiffness index (LASI), defined as the ratio of early diastolic transmitral flow velocity/lateral mitral annulus myocardial velocity $\left(E / e^{\prime}\right)$ to LA reservoir strain, was also calculated. LA reservoir strain has been shown to better correlate with LV filling than with maximal LA volume. LASI is a further parameter of atrial function, reflecting the change in pressure required to increase the volume of LA in a given measure. Recent studies have reported that LASI is a useful index to distinguish heart failure patients with preserved ejection fraction from those with asymptomatic diastolic dysfunction and is superior to maximal LA volume and LA reservoir strain in predicting elevated NT-proBNP levels in systemic sclerosis patients [9]. Furthermore, it has been documented that LASI is a powerful predictor of clinical outcomes in patients with reduced and mid-range ejection fraction [10]. The study by Zhao et al. adds further evidence on the clinical significance of LASI in the setting of uncomplicated essential hypertension by demonstrating that this index is independently correlated with LV mass index, arterial stiffness, and microalbuminuria. Of note, in multivariate analysis, the correlation was stronger for LV mass index and microalbuminuria than for arterial stiffness. LASI gradually increased from healthy controls throughout hypertensive patients with normal LA to those with LAE and LVH. It is worth noting that unlike hypertensive patients with structural alterations (i.e., LAE and LVH), those with normal atrial and ventricular structure exhibited LASI values that were not significantly different from those of normal subjects. This was not the case with the LA reservoir and conduit function, which was significantly impaired, even in hypertensive patients without LAE and LVH. This finding is of particular value considering that structural alterations of the atrium were limited to $30 \%$ of the total hypertensive population and significantly lower than the prevalence of LVH. Although a comment on the different prevalence of these two echocardiographic phenotypes is beyond the 
scope of this commentary, the fact that the diagnostic criterion for LAE does not take into account that the difference in sex (according to guidelines) deserves to be mentioned.

In conclusion, the study by Zhao et al. suggests that dysfunction of atrial mechanics precedes the development of both LAE and LVH and may be regarded as a very early sign of hypertension-mediated organ damage. At the moment, the same consideration cannot be made for LASI, whose clinical and prognostic significance in hypertensive patients without cardiac structural alterations merits further evaluation in future studies. Finally, it should be emphasized that the results of Zhao's study cannot be applied to the general hypertensive population, as they were collected in a hospitalized hypertensive cohort with elevated systolic blood pressure and suboptimal antihypertensive treatment.

\section{Compliance with ethical standards}

Conflict of interest The authors declare that they have no conflict of interest.

Publisher's note Springer Nature remains neutral with regard to jurisdictional claims in published maps and institutional affiliations.

\section{References}

1. Tarazi R, Miller A, Frohlich ED, Dustan HP. Electrocardiographic changes reflecting left atrial abnormality in hypertension. Circulation. 1966;34:818-21.
2. Miller JT, O'Rourke RA, Crawford MH. Left atrial enlargement: an early sign of hypertensive heart disease. Am Heart J. 1988; 116:1048-51.

3. Bombelli M, Facchetti R, Cuspidi C, Villa P, Dozio D, Brambilla $\mathrm{G}$, et al. Prognostic significance of left atrial enlargement in a general population: results of the PAMELA study. Hypertension. 2014;64:1205-11.

4. Gerdts E, Oikarinen L, Palmieri V, Otterstad JE, Wachtell K, Boman K, et al. Correlates of left atrial size in hypertensive patients with left ventricular hypertrophy. The Losartan Intervention For Endpoint reduction in hypertension (LIFE) study. Hypertension. 2002;39:739-43.

5. Cuspidi C, Rescaldani M, Sala C. Prevalence of echocardiographic left-atrial enlargement in hypertension: a systematic review of recent clinical studies. Am J Hypertens. 2013;26: 456-64.

6. Matsuda Y, Toma Y, Moritani K, Ogawa H, Kohno M, Miura T, et al. Assessment of left atrial function in patients with hypertensive heart disease. Hypertension. 1986;8:779-85.

7. Tadic M, Cuspidi C, Pencic B, Grassi G, Kocijancic V, Celic V. Left atrial phasic function in hypertensive patients with significant aortic stenosis and preserved ejection fraction. Hypertens Res. 2019;42:1200-8.

8. Zhao Y, Sun Q, Han J, Lu Y, Zhang Y, Song W, et al. Left atrial stiffness as a marker of early target organ damage in hypertension. Hypertens Res. 2020. https://doi.org/10.1038/s41440-020-00551-8. [Epub ahead of print].

9. Porpaczy A, Nogradi A, Vertes V, Tókes-Fuzesi M, Czirjak L, Komocsi A, et al. Left atrial stiffness is superior to volume and strain parameters in predicting elevated NT-proBNP levels in systemic sclerosis patients. Int J Cardiovasc Imaging. 2019;35: 1795-802.

10. Bytyçi I, Dini FL, Bajraktari A, Pugliese NR, D'Agostino A, Bajraktari G, et al. Speckle tracking-derived left atrial stiffness predicts clinical outcome in heart failure patients with reduced to mid-range ejection fraction. J Clin Med. 2020;9:1244. 